\title{
COMPARING PNEUMATIC AND WATER HYDRAULIC POSITIONING SYSTEM FOR PROCESS VALVES
}

\author{
Tapio VIRVALO \\ IHA, Tampere University of Technology, Finland \\ P.O.Box 589, 33101 Tampere \\ Tel. -358-3-31152262, Fax. -358-3-31152240 \\ e-mail tapio.virvalo@tut.fi
}

\begin{abstract}
Pneumatic positioning actuators are very commonly used in the control of process control valves in petroleum, chemist and paper industries. During the last decade several ideas have been presented how to use water hydraulics in different applications. Low-pressure water is available in some process industrial applications a little bit the same way as pneumatic power. Lately very demanding performance specification requirements have been presented for positioners of process valves. In this paper requirements set to control components of pneumatic and low pressure water hydraulic drives in order to fulfil these high quality performance specifications are studied. Different experimental tests have been done with both systems and results show that the specification can be fulfilled, but quite high performance components are required.
\end{abstract}

\section{KEYWORDS}

Positioning, pneumatic poison servo, water hydraulic position servo, process valves

\section{INTRODUCTION}

Pneumatic positioning actuators are very commonly used in the control of process control valves in petroleum, chemist and paper industries. Pneumatics has many advantages but also drawbacks in these applications. The main advantages are easy assembly and cost effectiveness and the most significant drawbacks are poor performance and large size.
During the last decade several ideas have been presented how to use water hydraulics in different applications. Applicability of water hydraulic servo systems in certain special applications has been also studied [1] [2] [3]. Low-pressure water is available in some process industrial applications a little bit the same way as pneumatic power. The performance requirements of pneumatic as well as low-pressure water hydraulic servo valves are studied in experimental tests. 
Typically special low cost and quite poor performance valves are used in the control of process valves. However, some very demanding requirements have been presented to the performance of process valves. The steady state and dynamic specifications of the positioning of process valves are presented in [4] which is a de facto standard in process industry. En Tech has specified three different specification levels. The parameter values of dynamics for the most demanding cases are; $\mathrm{Td}=0.1 \mathrm{~s}, \mathrm{~T} 86=0.4 \mathrm{~s}$ and $T s s=1 \mathrm{~s}$, Figure 1. In addition the following specifications are valid: dead band $0.06 \%$, step resolution $0.04 \%$, total hysteresis $0.1 \%$, and maximum overshoot $20 \%$ in the step size range $\pm 10 \%$.

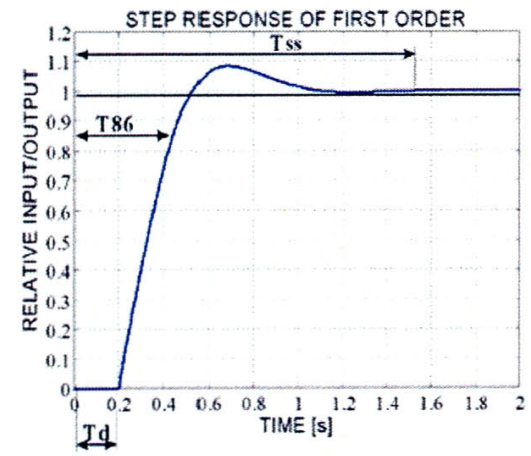

Figure 1. Performance criterion parameters

The studied application is shown in Figure 2 [5]. These kinds of positioning systems are used for instance in segment type process valves.

\section{GOALS OF STUDY}

The goals are to find if it is possible to achieve the specifications [4] with pneumatics and low pressure water hydraulics. The following goals are set:

* To find out if the highest performance criterions of process valve positioning system can be fulfilled with both systems

* To specify the specifications of the valid components of the system.

* To specify the performance requirements of the controller.

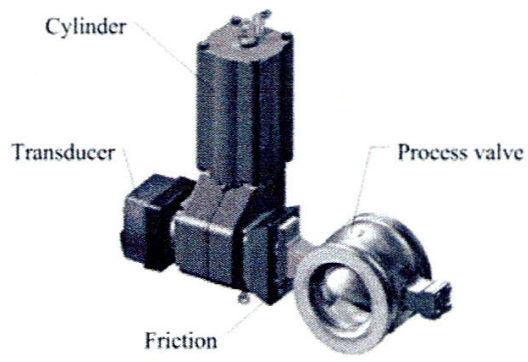

Figure 2. Process control valve (studied system)

\section{SPECIFICATIONS OF SYSTEMS}

A normal segment type process valve with standard pneumatic actuators is used in both cases, Figure 2. The rotation range of the segment is $90^{\circ}$. The control valve and position sensor are changed. In this study commercial pneumatic and water hydraulic servo valves are used.

The specifications of the pneumatic control system are as follows:

* Cylinder 125/20-76 (piston/rod-stroke [mm])

* Supply pressure 6bar

* Servo valve, nominal volume flow 7001/min, supply pressure 6bar, pressure drop/notch 1bar, hysteresis $<0.5 \%$, bandwidth $600 \mathrm{rad} / \mathrm{s}$

* Position sensor, incremental encoder, resolution $0.01 \%$

The specifications of the low pressure water hydraulic control system are as follows:

* Cylinder 80/20-76 (piston/rod-stroke [mm])

* Supply pressure 27 bar

* Servo valve, nominal volume flow 19l/min, pressure drop/notch 35 bar, hysteresis $<3 \%$, bandwidth $600 \mathrm{rad} / \mathrm{s}$

* Position sensor, differential capacitive angle sensor

* Analog-to-digital converter 14 bits.

The sampling time of the controller is $2 \mathrm{~ms}$ in both cases. Considering the performance specifications and load characteristics this application is very demanding for both positioning system. Inertia loads are remarkably low in these kinds of applications. Friction forces are high mostly because of the seals of the process valve. High friction forces make the specifications of resolution and 
hysteresis very challenging.

\section{EXPERIMENTAL TESTS}

Experimental tests are carried out both with pneumatic and pure water hydraulic systems. All components are commercial. The power supplies of both systems are taken from the main power units of the laboratory. The supply pressures are kept practically constant with an extra volume in the pneumatic supply line and an accumulator in the water hydraulic supply line.

\section{Initial tests}

In order to estimate fiction forces and the velocity gain of the systems some open loop measurements are carried out. As an example open loop responses of both systems are shown in Fig. 3 and 4. The maximum friction force is almost the same in both cases. The behaviour of the friction force in the pneumatic drive is more function of the piston position than in water hydraulic case. Remarkable difference is also in the relationship between the fiction force and the maximum force and the dynamic behaviour of the friction forces.

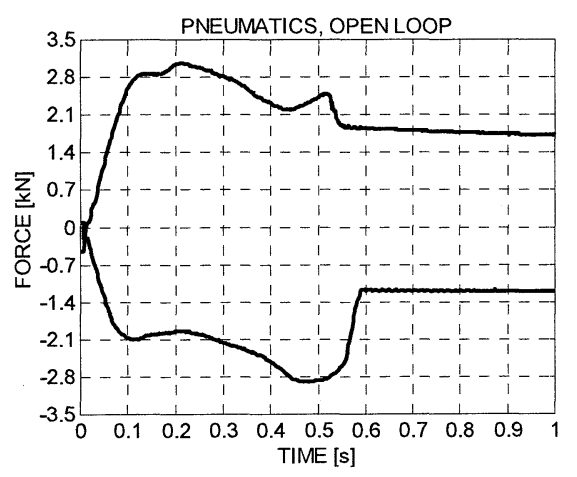

Figure 3. Measured friction force of pneumatic drive in both directions

The maximum friction force in pneumatic drive is about $45 \%$ and in water hydraulic drive about $22 \%$ of the maximum pressure forces. The pressure change rate is significantly lower in pneumatic drive than in water hydraulic drive in spite of that the maximum velocity of the pneumatic drive is significantly higher.

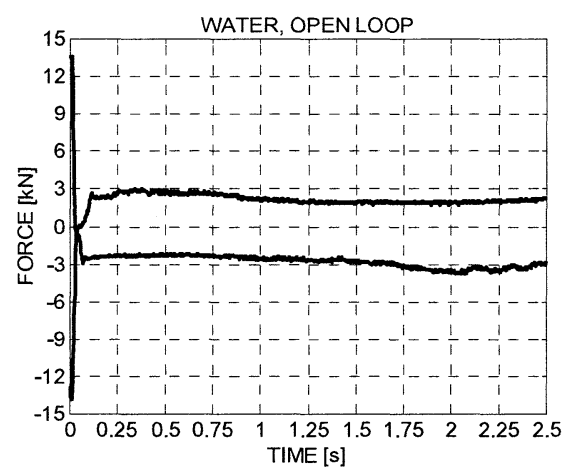

Figure 4. Measured friction force of water hydraulic drive in both directions

\section{Performance tests}

Because the specifications of the process valve positioner specify the performance of step responses, the following step responses are carried out. Figure 5 and 6 shows the position error responses of the pneumatic as well as water hydraulic drive, when the stroke length is about $10 \%$ corresponding about the $10 \mathrm{~mm}$ stroke of the cylinders.

Both cases fulfil well the performance specifications. The responses of the water hydraulic drive behave nicely and quite symmetrically, but there are some un-certainties in the responses of the pneumatic drive. A simple non-linear $\mathrm{P}$-controller is used in the water hydraulic case and a State Controller in the pneumatic case. Figure 7 and 8 shows responses of the position error of $1 \%$ strokes and Fig. 9 and 10 of $0.4 \%$ strokes, respectively.

All these responses fulfil the positioning accuracy and response time specifications. There are high frequency oscillations in responses of the water hydraulic drive, which are mostly due to the noise in the analogue position measuring system. The resolution of the position measuring system of the pneumatic drive can be clearly seen in the position error responses of Fig. 9. Because there is no overshoot in any one of the responses the tuning of the controllers might have been a little bit tighter. However the accuracy and response time requirements are fulfilled so there is no need to increase loop gains. 


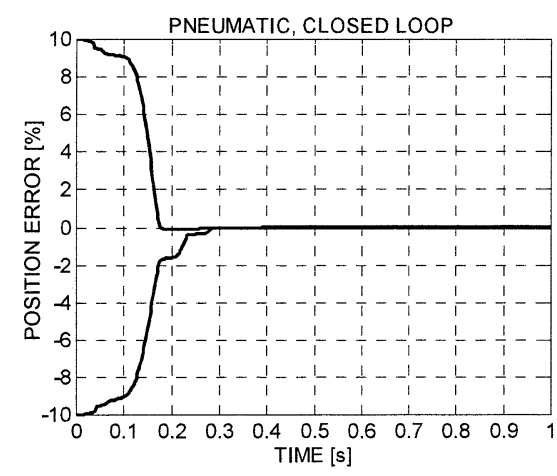

Figure 5. Measured position error responses, strokes $10 \%$, pneumatics

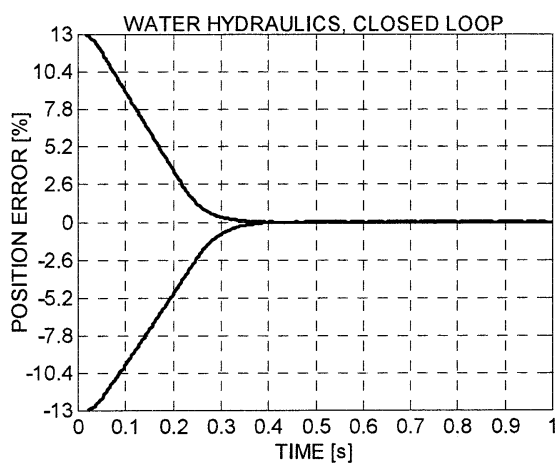

Figure 6. Measured position error responses, strokes $13 \%$, water hydraulics

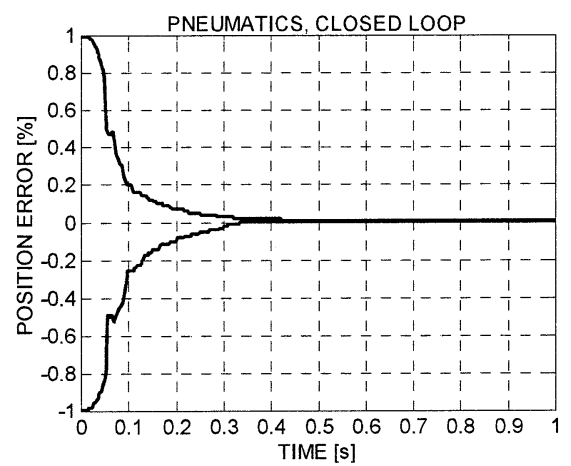

Figure 7. Measured position error responses, strokes $1 \%$, pneumatics

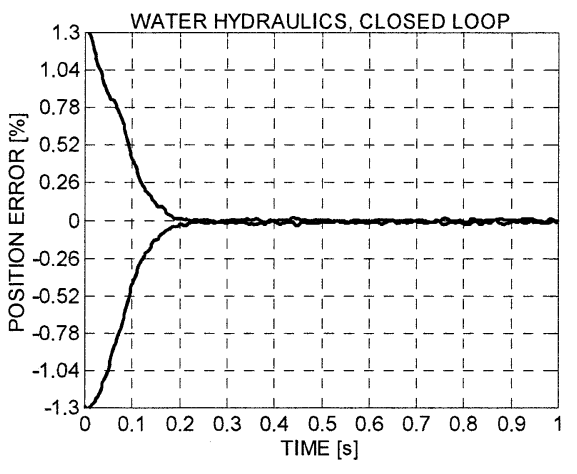

Figure 8. Measured position error responses, strokes $1.3 \%$, water hydraulics

The specifications presented in Introduction chapter, dead band $0.06 \%$, step resolution $0.04 \%$, total hysteresis $0.1 \%$, should be considered quite tough. In order to find those characteristics of both systems a continuous and discrete triangle shape wave reference signals have been used. As an example, the responses of the pneumatic drive to the continuous triangle wave are shown in Fig.11 and 12. The responses of the water hydraulic drive of discrete triangle wave are shown in Fig. 13 and 14, respectively. As can be seen both system fulfil the performance specifications.

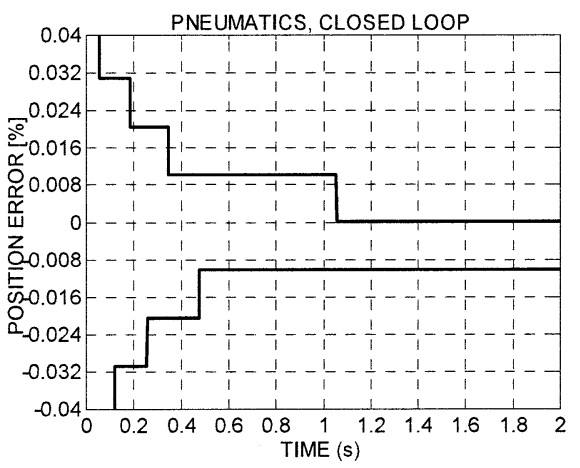

Figure 9. Measured position error responses, strokes $0.4 \%$, pneumatics 


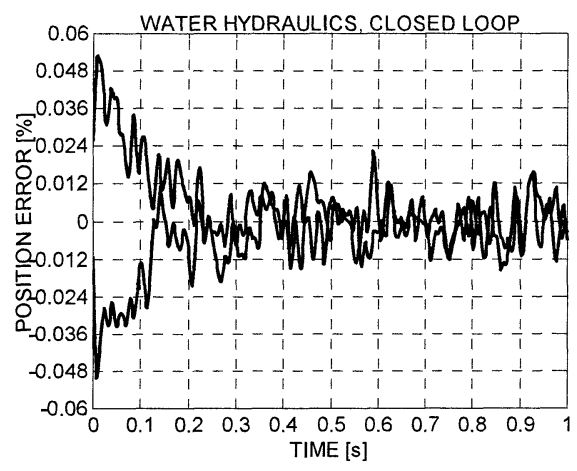

Figure 10. Measured position error responses, strokes $0.6 \%$, water hydraulics

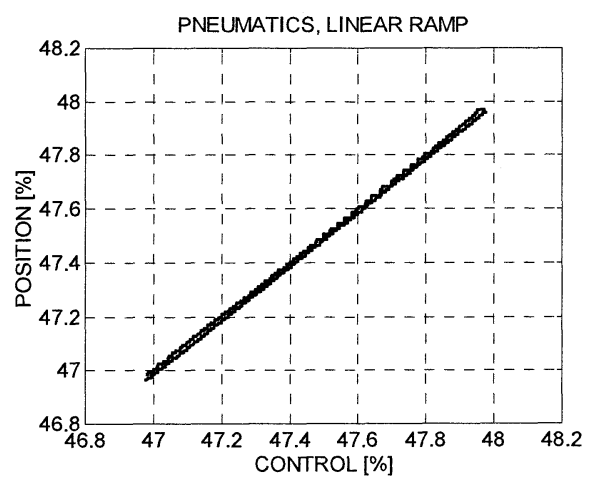

Figure 11. Measured position response of linear ramp (hysteresis), pneumatics

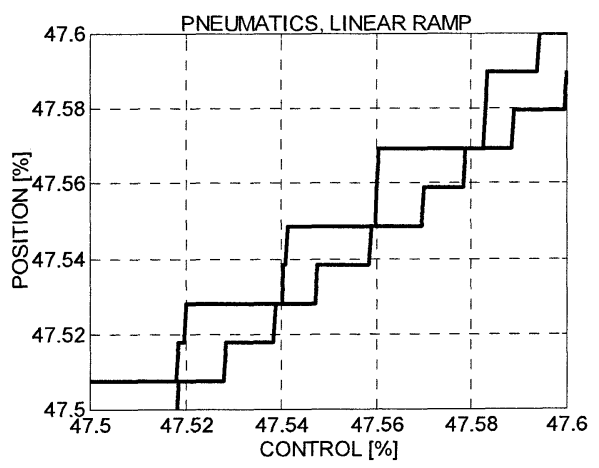

Figure 12. Zoomed measured position response of linear ramp (hysteresis), pneumatics

Differences in responses of two systems are mostly due to the used position sensors. There is significant noise in the position measurements of water hydraulic drive because of analogue position sensor. The incremental encoder is used in the pneumatic drive and no noise has been noticed.

\section{RESULTS COMPARED TO GOALS}

Both studied systems fulfil all specifications set by [4], as can be seen in Figures above. All specified steady state positioning accuracy, dead band, resolution, hysteresis and response times are reached without big problems. When the zero point of valves is well tuned no drift or hunting takes place.

The most critical component in both cases is the servo valve. The servo valves used in this study are good quality commercial valves. Their size is quite suitable with the used supply pressures. The problem is their costs. These kinds of commercial servo valves, especially water hydraulic servo valve, are too expensive for these applications. The specified positioning accuracy, resolution, dead band and hysteresis require reasonable good position measurement system. The resolution should be at least around $0.01 \%$, which means problems with analogue position sensors.

Because the maximum velocity of the cylinder is relatively low the high position loop gains can be used and so a non-linear P-controller is enough in the water hydraulic case. The pneumatic drive requires at least a State Controller. This not a big problem, because high resolution position feedback is needed anyway and so the required velocity and acceleration can be achieved by differentiate from position signal. The required sampling time is $<10 \mathrm{~ms}$.

As a summary it can be said that with relatively high quality commercial components the highest specifications of steady state and dynamic behaviour set by [4] can be realized with pneumatics as well as with low pressure water hydraulics. 


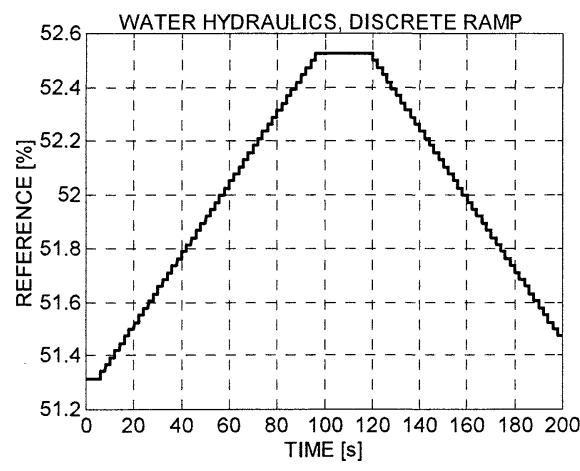

Figure 13. Measured discrete ramp, step $0.022 \%$, water hydraulics

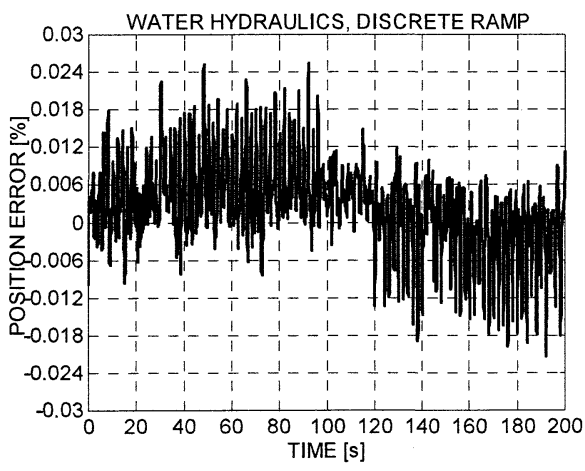

Figure 14. Measured position error, discrete ramp, water hydraulics

\section{Discussion}

In order to fulfil the performance specifications the steady state characteristics of servo valves should be around the specifications of the servo valve used in this study. Electric power of process valve control systems is very limited (around $5-10 \mathrm{~mW}$ ) which also might limit dynamic properties of servo valves. Some experimental tests are realized by using a second order low-pass filter in the control signal of the servo valves in order to reduce the dynamics of the servo valve. When the bandwidth of the filter is one tenth of the bandwidth of the servo valves and the damping lower $(\delta=0.5)$ the overall behaviour of both systems do not differ remarkably from original behaviour. However, a State Controller is also needed in the water hydraulic drive and the controller the pneumatic drive has to be re-tuned.

\section{CONCLUSIONS}

According to this study the following conclusions can be made.

* Highest steady state and dynamic performance specifications of positioners of process valves can be realized both with pneumatic and low pressure water hydraulic drives using commercial servo valves

* High steady state performance of servo valves is required in both cases. Low hysteresis is the most important.

* Dynamics of servo valves is not very critical. It could be one tenth of commercial servo valves.

* Commercial servo valves are too expensive for these applications, especially water hydraulic servo valves.

* Quite high resolution of position sensor is required. Resolution should be at least around $0.01 \%$ of the full stroke of positioners.

\section{REFERENCES}

1. Vuorisalo, M. \& Virvalo, T. 2000. Comparing the control methods of fast water hydraulic on/off-valves in pressure control. The 7th Mechatronics Forum, 6th-8th September, 2000, Atlanta, Georgia, USA. 5 p.

2. Vuorisalo, M. \& Virvalo, T. 2000. Smart material actuators for fluid power valves with different pressure mediums. 9th Annual Conference on MMV, 19th-21st, September, 2000, Harvey Bay, Queensland, Australia. p. 305-313.

3. Vuorisalo, M., Virvalo, T. \& Anttonen, P. 2001. Different types of pilot stages for a water hydraulic control valve. ICFP12001 April 3-5, 2001, Hangzhou, China. p. 435-439.

4. EnTech: Control Valve Dynamic Specifications, 1998.

5. Metso Automation, 3 R 20EN Issue $8 / 2001$ 